\title{
Enhanced effect of photodynamic therapy in ovarian cancer using a nanoparticle drug delivery system
}

\author{
ZHAO LI ${ }^{1}$, LIPING SUN ${ }^{1}$, ZAIJUN LU ${ }^{2}$, XUANTAO SU ${ }^{3}$,

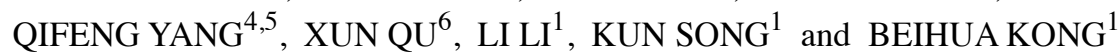 \\ ${ }^{1}$ Department of Obstetrics and Gynecology, Qilu Hospital, ${ }^{2}$ School of Chemistry and Chemical Engineering, Shandong \\ University, Jinan, Shandong 250012; ${ }^{3}$ Department of Biomedical Engineering, School of Control Science and Engineering, \\ Shandong University, Jinan, Shandong 250061; ${ }^{4}$ Department of Breast Surgery, Qilu Hospital, Shandong University, \\ Jinan, Shandong 250012, P.R. China; ${ }^{5}$ The Cancer Institute of New Jersey, Robert Wood Johnson Medical School, \\ New Brunswick, NJ 08903, USA; ${ }^{6}$ Department of Basic Medicine, Shandong University, Jinan, Shandong 250012, P.R. China
}

Received May 6, 2015; Accepted June 29, 2015

DOI: 10.3892/ijo.2015.3079

\begin{abstract}
Nanoparticles are promising novel drug delivery carriers that allow tumor targeting and controlled drug release. In the present study, we prepared poly butyl-cyanoacrylate nanoparticles (PBCA-NP) entrapped with hypocrellin B (HB) to improve the effect of photodynamic therapy (PDT) in ovarian cancer. An ovarian cancer ascites model using Fischer 344 rats and PBCA-NP entrapped with HB (HB-PBCA-NP) were formed successfully. The pharmacodynamic characteristics and biodistribution of the HB-PBCA-NP system were evaluated by comparison with HB dimethyl sulfoxide (HB-DMSO) and testing at various time-points following intraperitoneal drug administration. HB-PBCA-NP-based PDT combined with cytoreductive surgery was then administrated to the tumor-bearing animals. Kaplan-Meier survival analysis was performed to assess the therapeutic effect of the nanoparticle system. The serum HB concentration peaked $4 \mathrm{~h}$ after drug administration in the nanoparticle system, and $1 \mathrm{~h}$ with HB-DMSO. The peak exposure time of tumor tissues was also extended (4 vs. 2 h), and PBCA-NP remained present for much longer than HB-DMSO. Although PDT combined with surgery prolonged the survival time significantly compared with surgery alone ( 84 days, $\mathrm{P}<0.05$ ), there was no significant
\end{abstract}

Correspondence to: Professor Kun Song, Department of Obstetrics and Gynecology, Qilu Hospital, Shandong University, 107 Wenhua Xi Road, Jinan, Shandong 250012, P.R. China

E-mail: songkun2001226@163.com

Abbreviations: PDT, photodynamic therapy; HB, hypocrellin B; PBCA, poly(butyl-cyanoacrylate); BCA, $\alpha$-butyl-cyanoacrylate; NP, nanoparticles; TEM, transmission electron microscopy; DLS, dynamic light scatting; HPLC, high performance liquid chromatography; EPR, enhanced permeation and retention; THF, tetrahydrofuran; AUC, area under the curve; RES, reticuloendothelial system

Key words: controlled release, nanoparticles, ovarian cancer, photodynamic therapy, tumor targeting difference in the survival time of animals that received either HB-PBCA-NP- or HB-DMSO-based PDT after cytoreductive surgery (99 vs. 95 days, $\mathrm{P}=0.293$ ). PBCA-NP exhibited potential advantages in controlled drug release and tumor targeting, which was beneficial for HB-based PDT. PDT combined with surgery prolonged the survival time, suggesting that this might be an alternative treatment option for ovarian cancer.

\section{Introduction}

Ovarian cancer is responsible more than half of the deaths caused by gynecological malignancies (1). It is a highly metastatic disease that is rarely diagnosed when it is confined to the ovaries due to a lack of characteristic symptoms (2). Peritoneal metastasis is the principal cause of death in patients with advanced or recurrent ovarian tumor (3-5). Even aggressive peritoneal or cytoreductive surgery leave large areas of the peritoneal cavity untreated (6). Although chemotherapy is a traditional adjuvant choice, experience over the past few decades has suggested that it does not enhance patient survival. Therefore, a novel adjuvant therapy is needed urgently.

Photodynamic therapy (PDT) is a promising alternative to the conventional therapeutic strategies for the management of intraperitoneal carcinomas (7). PDT is based on the delivery of a photosensitizer followed by exposure to light of a specific wavelength, which converts oxygen into reactive singlet oxygen $\left({ }^{1} \mathrm{O}_{2}\right)$. This causes selective cancer cell death, because cancer cells uptake more photosensitizers than do normal cells $(8,9)$. Singlet oxygen eliminates tumor tissue by causing microvascular acute injury, blocking vessels and also inducing cancer cell apoptosis (10-12). Another interrelated mechanism of the antitumor effects of PDT is the induction of a local inflammatory reaction that leads to the development of systemic immunity (13).

Hypocrellin B (HB) is a traditional Chinese drug that is extracted from fungus (Hypocrella bambuase) of a Chinese herb; it is an excellent natural photosensitizer. In China, it is commonly used to treat rheumatoid arthritis, and gastric and skin diseases (14). Several studies have suggested that it is a 
promising non-porphyrin photosensitizer, because it causes high singlet oxygen quantum yields, has a low tendency for aggregation and rapid metabolism in vivo (15).

The development of nanotechnology has introduced more choices for cancer treatment. Nanoparticle delivery systems allow specific tumor targeting. The leaky vasculature in tumor tissues promotes the uptake of nanoformulations, and the impaired and poor lymphatic drainage traps nanosized particles inside tumor tissues $(16,17)$ in the so-called 'enhanced permeation and retention' (EPR) effect. Therefore, drugs encapsulated in nanoparticles could accumulate at high levels in tumor tissues for a longer time. Studies have shown that nanoparticles carrying anticancer agents prolonged drug retention in tumors, which could inhibit tumor growth (18-20) and improve the efficiency of cancer therapy (17). The materials used for nanoparticle preparation are often biodegradable; therefore, the drugs they encapsulate can be released gradually and then degraded slowly. This ensures that the drug concentrations in target tissues remain high. Recent studies have revealed that poly(butyl-cyanoacrylate) nanoparticles (PBCA-NPs) are effective drug carriers $(21,22)$. In the present study, we synthesized PBCA-NP encapsulated with HB to perform tumor-targeted PDT in ovarian cancer-bearing Fischer 344 rats.

\section{Materials and methods}

Preparation of HB-PBCA-NP. Dextran-70 and Pluronic F-127 (Sigma-Aldrich, St., Louis, MO, USA) were dissolved in $5 \mathrm{ml}$ distilled water and the $\mathrm{pH}$ was adjusted to 1.5. The solution was mixed with $\alpha$-butyl-cyanoacrylate (BCA; Shunkang Pharmaceutical Co., Ltd., Beijing, China) by magnetic stirring at $600 \mathrm{rpm}$ for $4 \mathrm{~h}$ at $25^{\circ} \mathrm{C}$, and the $\mathrm{pH}$ was then raised to 7.0. The solution was stirred for another $30 \mathrm{~min}$ until polymerization was complete. The filtered nanoparticles were kept at $4^{\circ} \mathrm{C}$ for $12 \mathrm{~h}$. Then, $2.5 \mathrm{ml} \mathrm{HB}$ (Chinese Academy of Science, Beijing, China) in ethanol $(0.5 \mathrm{mg} / \mathrm{ml})$ was added to nanoparticle solution and sonicated. The suspension was then incubated for an additional $12 \mathrm{~h}$ at $4^{\circ} \mathrm{C}$ and vacuum-freeze dried to obtain HB-PBCA-NP. The size and other features of the particles were tested using laser dynamic light scatting (DLS; Malvern Instruments Ltd., Worcestershire, UK) and transmission electron microscopy (TEM, Tecnai G2 F20 S-TWIN; FEI Co., Hillsboro, OR, USA).

Cell line and animals. The poorly differentiated Fischer 344 rat-derived epithelial ovarian cancer line NuTu-19 was maintained in the Basic Medicine Laboratory of Qilu Hospital. Cells were cultured in Dulbecco's modified Eagle's medium (DMEM; Sigma) supplemented with 10\% heat-inactivated FCS (Gibco-Life Technologies, Grand Island, NY, USA), and incubated under standardized conditions $\left(37^{\circ} \mathrm{C}, 5 \%\right.$ carbon dioxide and $100 \%$ humidity). NuTu-19 cells were harvested using $0.25 \%$ trypsin and $0.02 \%$ EDTA, and were washed twice with PBS. All experiments were performed using exponentially growing cells.

Animal model. Pathogen-free female Fischer 344 rats (Beijing Weitong Lihua, Beijing, China), weighing 100-120 g, were housed in a pathogen-free animal facility and given a standard laboratory diet and water. A total of $2 \times 10^{6}$ cells were injected into the peritoneal cavity of each rat. The temperature of the cage was kept at $22 \pm 2^{\circ} \mathrm{C}$, and relative humidity was kept between $45-65 \%$. The present study was performed in strict accordance with the Guide for the Care and Use of animals of Qilu Hospital, Shandong University, China. The protocol was also approved by Ethics Committee of Animal Experiments of Qilu Hospital of Shandong University. The experiment was performed in accordance with the Guide for the Care and Use of Laboratory Animals. The animals were then observed daily and weighed twice a week. Sodium pentobarbital was injected intraperitoneally before surgery and all efforts were made to minimize suffering.

Pharmacokinetic and distribution of $H B$ in animals. HB-PBCA-NP and HB were freshly prepared prior to use by dissolving in normal saline and DMSO, respectively. Tumorbearing Fischer 344 rats were divided into two groups, which received $10 \mathrm{mg} / \mathrm{kg}$ of either HB-DMSO or HB-PBCA-NP by intraperitoneal injection. Blood samples were then collected from the postcava vessel at various time-points $(10,30$, $40 \mathrm{~min}, 1,2,4,6,12$ and $24 \mathrm{~h}$ ). All blood samples were coagulated for $2 \mathrm{~h}$ at room temperature, and were then centrifuged at $2 \times 10^{3} \mathrm{~g}$ to separate the serum. The rats were sacrificed $1,2,4,6,12$ and $24 \mathrm{~h}$ after drug injection, and their tissues (liver, spleen, lung, kidney, small intestine, uterus and tumor) were harvested. Two hundred microliters of tetrahydrofuran (THF; Shiyou Biotech Co., Ltd., Tianjin, China) was added to the serum and minced tissue samples, which were then centrifuged again. The supernatants were analyzed using high performance liquid chromatography (HPLC; Agilent Technologies, Palo Alto, CA, USA). The concentration of $\mathrm{HB}$ in the different samples was determined at various timepoints according to the areas under the curve. The data were processed using DAS (drug and statistics for Windows) to obtain pharmacokinetic parameters.

PDT in vivo. The tumor-bearing rats were divided randomly into four groups 6 weeks after inoculation. The control groups included untreated animals (group A) and those that received only cytoreductive surgery (group B). Animals in group $\mathrm{C}$ received cytoreductive surgery and PDT followed by intraperitoneal injection with $10 \mathrm{mg} / \mathrm{kg}$ HB-DMSO. Group D received surgery and PDT followed by intraperitoneal injection with $10 \mathrm{mg} / \mathrm{kg}$ HB-PBCA-NP. The PDT was performed when the concentration of drug reached a peak in tumor tissues. Food was removed from the cages $12 \mathrm{~h}$ before the operation, and rats were anesthetized using $40 \mathrm{mg} / \mathrm{kg}$ sodium pentobarbital (Sigma). An incision $\sim 5 \mathrm{~cm}$ in length was made on the abdominal wall to fully expose the organs in the abdominal cavity. Cytoreductive surgery was then performed to remove the maximum amount of tumor lesions. The laser output energy delivered to the surface of the peritoneal cavity of each rat was $50 \mathrm{~J} / \mathrm{cm}^{2}$ with total energy of $200 \mathrm{~mW}$. During laser irradiation, warm normal saline was dripped into the peritoneal cavity to reduce the loss of body fluid. The operations were performed under sterile conditions, and the animals were kept warm with a heating device until they awoke. All animals were then followed, and survival data were collected for further analysis. 
A

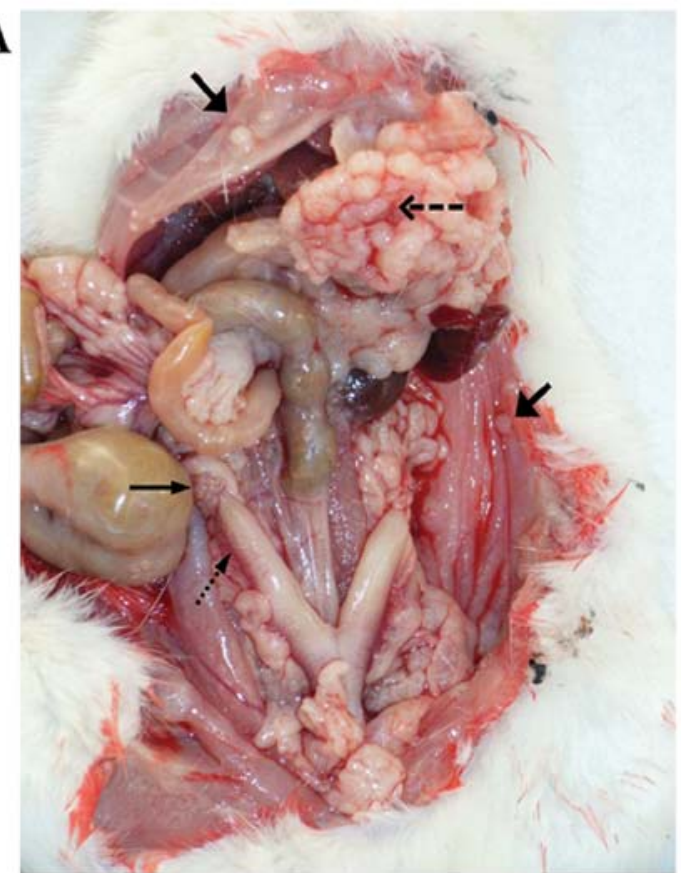

B

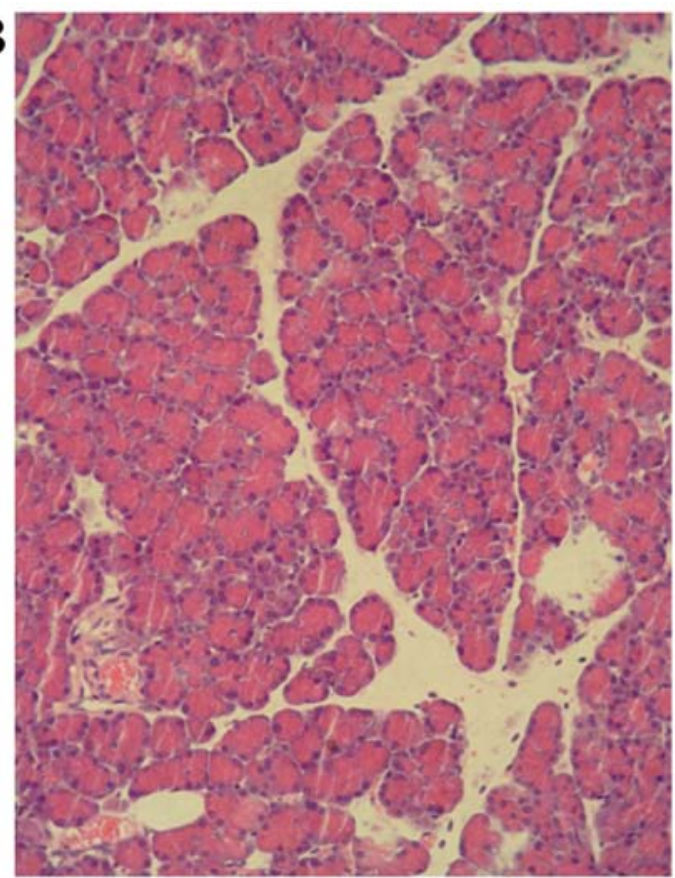

Figure 1. The anatomical structure of rats. (A) The indicated tissues are tumor nodules $(\rightarrow)$, ovary $(\rightarrow)$, uterus $(--\bullet)$ and omentum $(---)$. The pathological section of tumor nodules in peritoneal cavity of rat. (B) $\mathrm{H} \& \mathrm{E}$ staining shows the tissue is adenoid structure. The glands arrange closely and the eosinophil part in the center part of gland is secretion.

Statistical analysis. Data were analyzed using SPSS 13.0 for Windows (SPSS) software. The significance of differences among groups was analyzed using two-way ANOVA and Student-Newman-Keuls q-test. Survival studies were assessed using Kaplan-Meier survival analysis. $\mathrm{P}<0.05$ was used to indicate statistical significance.

\section{Results}

Features of nanoparticles. The mean size of PBCA-NP was $95 \mathrm{~nm}$ in diameter (range, 30-200 nm). TEM pictures revealed that the nanoparticles were well separated, and spherical in appearance. The drug encapsulation efficiency was $92.7 \%$ and drug-loading content was $15 \%$ for PBCA-NP; therefore, $\mathrm{HB}$ was efficiently encapsulated into the nanoparticles. The HB-PBCA-NPs were adequate for use in subsequent experiments.

Fischer 344 rat tumor model. Six weeks after the injection of NuTu-19 cells, all rats developed ovarian tumors in their abdominal cavity. A large number of cancer nodules appeared on the surface of the peritoneum, omentum, diaphragm, bowel, and reproductive organs. The images presented in Fig. 1A show the nodules and principal tissues in the abdominal cavity. Malignant bloody ascites were also detected in the abdominal cavity. Pathological analysis of tumor tissue sections confirmed the presence of adenocarcinoma (Fig. 1B).

Pharmacokinetic parameters. Pharmacokinetic parameters were calculated to assess the controlled drug release tendency of the NP. The half-lives of HB in the blood delivered using HB-DMSO and HB-PBCA-NP was 9.45 and $12.99 \mathrm{~h}$, respectively. The area under the curve (AUC) of HB-PBCA-NP

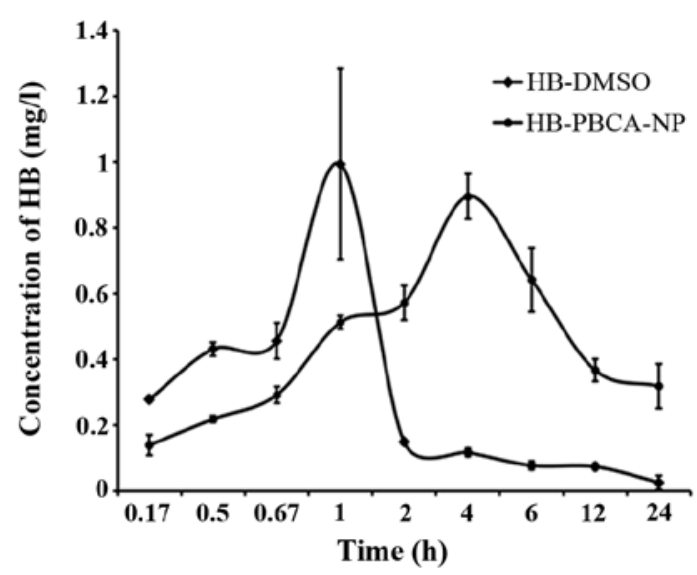

Figure 2. Serum distribution of $\mathrm{HB}$ at different time-points after delivery using the two systems.

was $10.42 \mu \mathrm{g} / \mathrm{ml} / \mathrm{h}$, which was larger than that of HB-DMSO $(2.60 \mu \mathrm{g} / \mathrm{ml} / \mathrm{h})$. The time-to-peak was 1 and $4 \mathrm{~h}$ for HB-DMSO and HB-PBCA-NP, respectively. These data confirmed that HB carried by nanoparticles was cleared more slowly than HB-DMSO.

Distribution of $H B$ in vivo. Serum HB levels increased rapidly in the HB-DMSO group. The highest concentration was $0.99 \mathrm{mg} / \mathrm{l}$, which was reached at $1 \mathrm{~h}$ after injection. However, very little $\mathrm{HB}$ was detected at $12 \mathrm{~h}$ after injection. However, HB levels in the serum of the HB-PBCA-NP group increased much slower. The highest concentration $(0.8954 \mathrm{mg} / \mathrm{l})$ was reached $4 \mathrm{~h}$ after injection. More importantly, the HB concentrations in the serum remained relatively high $(0.3187 \mathrm{mg} / \mathrm{l})$ after $12 \mathrm{~h}$ compared with the HB-DMSO group (Fig. 2). 

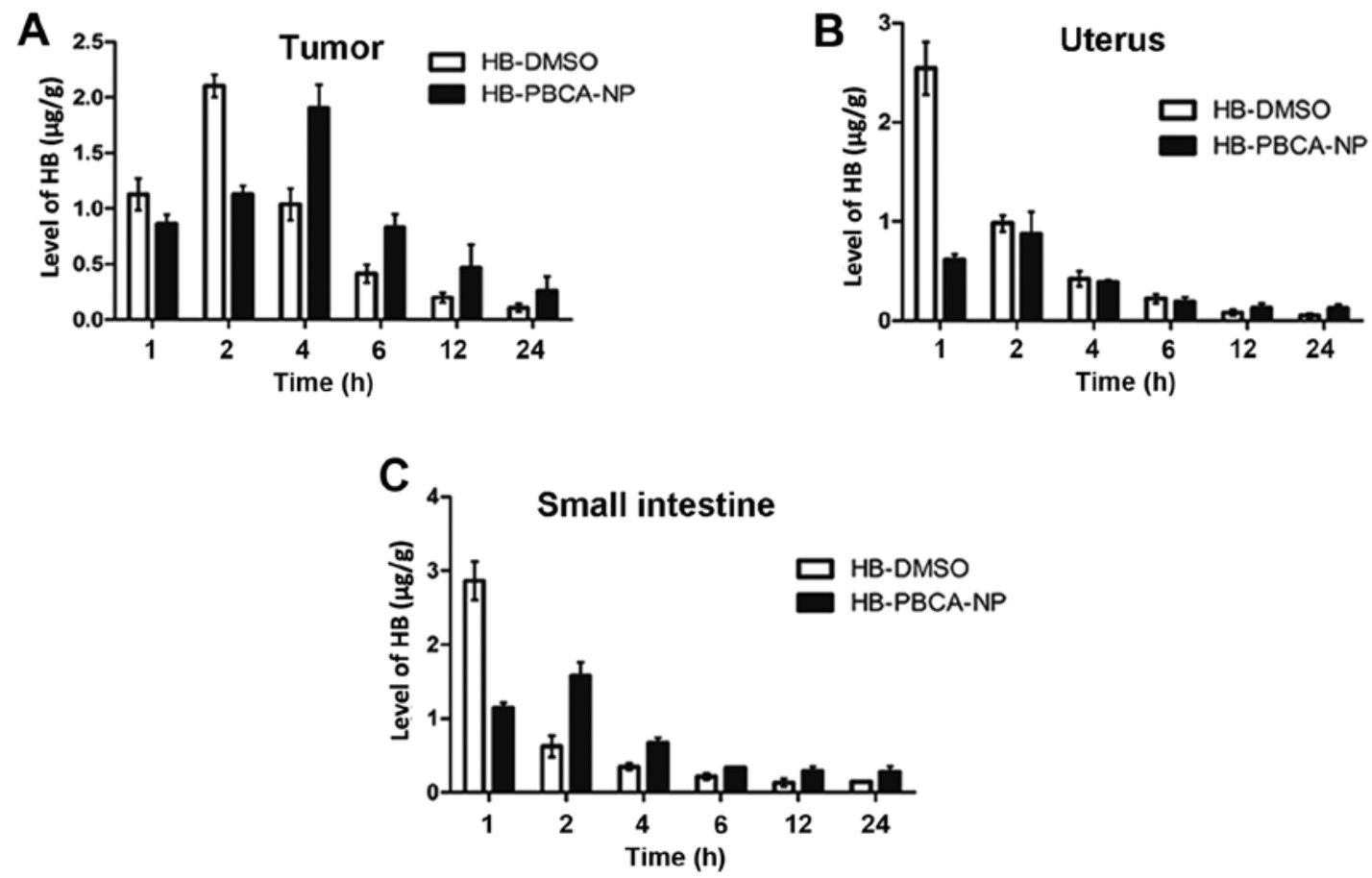

Figure 3. The HB distribution in different tissues at each time-point. The HB-DMSO gathered more rapidly in tissues than HB-PBCA-NP. The levels of HB-PBCA-NP in tumor reached a peak $4 \mathrm{~h}$ after injection (A), which was higher than (B) in the uterus and (C) the small intestine.
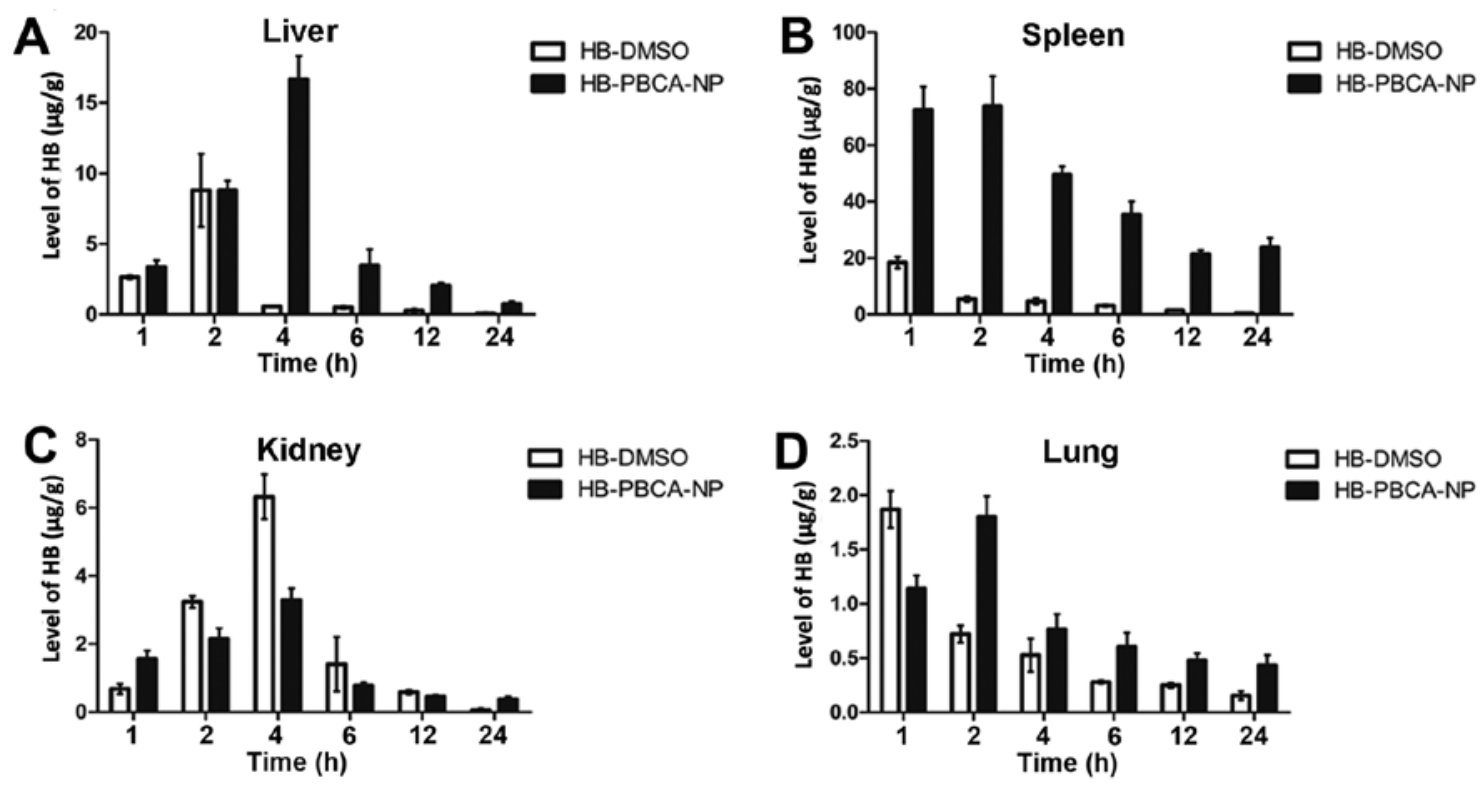

Figure 4. The levels of HB in two delivery methods for (A) liver, (B) spleen, (C) kidney and (D) lung. The HB-PBCA-NP was greatly enriched in the liver and spleen, while at low level in the kidney.

The levels of HB in each tissue at various time-points after treatment are shown in Figs. 3 and 4. The peak time at which the maximal concentration of HB was obtained was delayed in the HB-PBCA-NP group in all tissues compared with the HB-DMSO group. For example, the peak time in tumor tissue (Fig. 3A) and the liver (Fig. 4A) was 4 and $2 \mathrm{~h}$ in HB-PBCA-NP and HB-DMSO groups, respectively. The concentrations in the uterus (Fig. 3B), small intestine (Fig. 3C), spleen (Fig. 4B) and lung (Fig. 4D) peaked at $2 \mathrm{~h}$, which was later than in the HB-DMSO group $(1 \mathrm{~h})$. An additional important finding was that HB levels in HB-PBCA-NP remained higher than DMSO group in all tissues even $24 \mathrm{~h}$ after drug administration (Figs. 3 and 4). For example, the levels of HB in tumors in the HB-PBCA-NP group $24 \mathrm{~h}(0.26 \pm 0.13 \mu \mathrm{g} / \mathrm{g})$ were much higher than in the HB-DMSO group $(0.11 \pm 0.04 \mu \mathrm{g} / \mathrm{g})$. In addition, HB levels were higher in all tissues in the HB-PBCA-NP compared with the HB-DMSO group, $4 \mathrm{~h}$ after drug administration except for the uterus and kidney (Fig. 4C). Fig. 4 demonstrates that the spleen (Fig. 4B) and liver (Fig. 4A) of tumor-bearing rats had the highest concentration of HB, followed by the kidney 

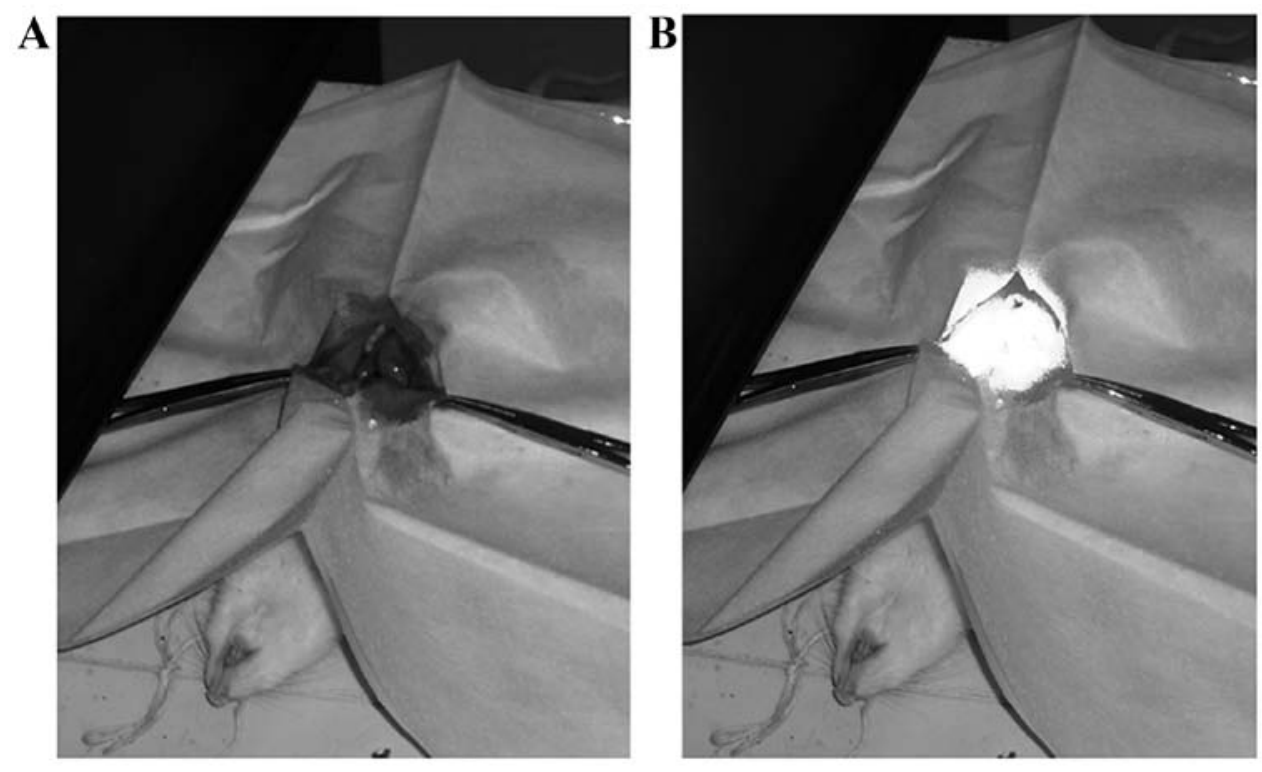

Figure 5. Photodynamic therapy applied on a rat. (A) The rat receiving cytoreductive surgery with fully exposed intraperitoneal cavity. (B) The rat receiving laser irradiation.

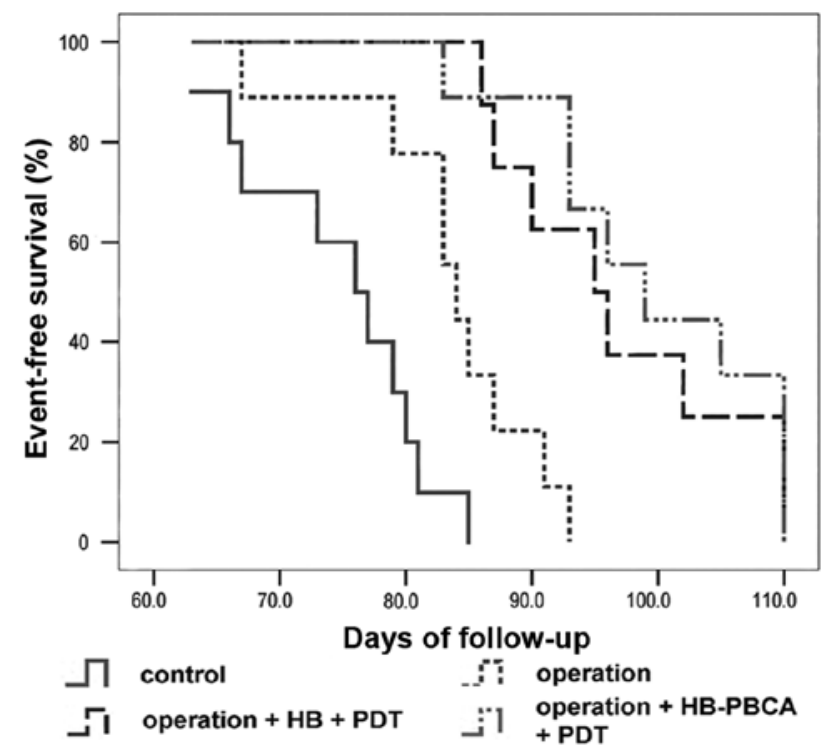

Figure 6. Kaplan-Meier survival curves for tumor-bearing Fischer 344 rats. The survival time of two groups (animals that received no treatment and cytoreductive surgery alone) compared with the two treatment groups (surgery and PDT).

(Fig. 4C) and lung (Fig. 4D). The spleen captured a large number of PBCA-NP (four-times more than the liver). Notably, the levels of $\mathrm{HB}$ in the liver and spleen $4 \mathrm{~h}$ after injection in HB-PBCA-NP group were $16.6 \pm 1.67$ and $49.62 \pm 2.952 \mu \mathrm{g} / \mathrm{g}$, respectively, compared with $0.57 \pm 0.02$ and $4.68 \pm 1.17 \mu \mathrm{g} / \mathrm{g}$ in the HB-DMSO group. The difference of the highest levels of HB in other tissues was not as great as in the two groups.

Efficacy of PDT. The images of PDT process are shown in Fig. 5. Four rats died within 10 days of surgery as a result of the invasive operation. These animals were excluded from the survival analysis. All animals were followed up until death. Kaplan-Meier survival curves (Fig. 6) revealed that the median survival time of groups A and B was 76 (95\% CI, 69.8-82.2 days) and 84 days (95\% CI, 81.08-86.92 days), respectively. Rats in both groups showed progressive cachexy. The median survival time of groups C and D was 95 (95\% CI, 86.68-103.316) and 99 days (95\% CI, 90.24-107.74), which were both longer than the control groups. Surgery alone improved animal survival, whereas surgery combined with PDT could more effectively extend survival time. These difference in survival time when each group was compared with the others individually was significant $(\mathrm{P}<0.05)$, except for groups $\mathrm{C}$ and $\mathrm{D}(\mathrm{P}=0.293)$.

\section{Discussion}

Two thirds of patients with ovarian cancer have already developed peritoneal carcinomatosis at diagnosis (23). The curative rate decreases substantially, once the disease has metastasized to the pelvic organs, the abdomen or beyond the peritoneal cavity (2). PDT can destroy cancerous tissue selectively, sparing normal tissue (24). Therefore, it might be a choice for the treating the superficial or microscopic lesions that remain after debulking surgery in ovarian cancer. The feasibility of this approach was demonstrated in clinical studies $(6,25)$.

Nanoparticle delivery systems allow tumor targeting and prolonged drug release. Therefore, we hypothesized that PBCA-based nanoparticle systems carrying the photosensitizer HB could enhance the efficiency of PDT. HB is an excellent second-generation natural photosensitizer that is extracted from a parasitical fungus in China. However, the clinical use of natural HB is severely restricted by its poor water solubility (15). In the present study, HB was captured inside PBCA NP to develop a water soluble HB-PBCA-NP system that could be used directly. Importantly, the HB-PBCA-NP system significantly delayed the clearance of HB from rat serum. The peak time was extended from 1 to $4 \mathrm{~h}$ by the injection of HB-PBCA-NP. Accordingly, the half-life of HB in the NP group was also prolonged from 9.45 to $12.99 \mathrm{~h}$. Twenty-four hours after injection, HB remained detectable in the serum 
of the NP group at relatively high concentrations, which was important for achieving a minimum effective concentration of HB. The area under the curve of HB in the NP particle group was also larger than control, meaning a higher bioavailability of the drug. An ideal drug delivery formulation should release the drug at a minimum effective concentration over a longer period of time to achieve maximum efficiency (17). In the present study, the HB-PBCA-NP system achieved all these characteristics.

Similar to the serum metabolism, the peak time of HB in the NP group was delayed in tissues. The peak time in tumor tissue was 4 and $2 \mathrm{~h}$ in the NP and HB-DMSO groups, respectively. PDT was administered when HB levels reached their peak. The concentration in other tissues, particularly in the small intestine and uterus, was lower. This helped to achieve the optimal therapeutic effect using a minimum dose of laser. This helped reduce internal damage, such as perforation of the small intestine. HB levels remained high in the NP group $24 \mathrm{~h}$ after drug administration, which would theoretically allow PDT to be repeated.

The HB-PBCA-NP system also altered the biodistribution of HB. The spleen and liver absorbed more HB than the other tissues in NP group. In the DMSO group, there was no significant difference in the HB levels among tissues. The spleen and liver are both reticuloendothelial system (RES) organs that capture foreign materials entering the body. In the present study, HB-PBCA-NPs were delivered intraperitoneally rather than intravenously; therefore, they were captured in large numbers by the spleen and liver. To evade the RES, nanoparticles should be further modified on their surface. To achieve this, we have synthesized an addition type of NP that is modified by folate on the surface, making it suitable for intravenous applications (unpublished data). The maximum concentration of $\mathrm{HB}$ in the kidneys of the HB-PBCA-NP group was lower than in the HB-DMSO group. Therefore, most PBCA-NP decomposed in the body rather than being excreted by the kidney.

The Kaplan-Meier survival curves revealed that both HB-DMSO- and HB-PBCA-NP-based PDT could significantly prolong animal survival compared with control. This could be attributed to the killing effect of PDT on the residual nodules that could not be removed surgically. We demonstrated previously that PDT could efficiently reduce the volume of subcutaneous tumors in nude mice using hemoporfin (26). PDT was performed after surgery without causing excess injury to the animals. The surgical procedure also allowed an adequate operating area for the application of PDT. However, there was no statistical difference in survival time between group $\mathrm{C}$ and $\mathrm{D}$. There were many factors that could have influenced this observation. The unavoidable capture of NP by the RES might reduce the concentration of $\mathrm{HB}$ in the tumor tissues. Therefore, the nanoparticles need to be modified further and be made small enough to achieve maximum targeting to the tumor tissue. Although the levels of HB in the DMSO group were higher than those in the NP group, this difference was not significant, and perhaps contributed little to the effect of PDT in vivo. In addition, photochemical oxygen consumption might overwhelm the oxygen in the microvasculature during PDT (27) which might also influence the outcome of the PDT.
A good animal model is essential for medical research. Immunodeficiency mice are commonly used for tumor-based experiments, because they can be used as tumor-bearing animal models. However, this kind of mouse was not suitable for our current study because of their susceptibility to microbes. As such, we needed a model that is strong enough to undergo the surgical and PDT procedures. We selected the Fischer 344 rat, which has a normal immune system, to build an ovarian cancer model. The NuTu-19 cell line is derived from Fischer 344 rat poorly differentiated papillary serous ovarian adenocarcinoma. Pathological analysis confirmed that all rats had developed ovarian adenocarcinoma after the intraperitoneal injection of NuTu-19 cells. The progressive tumor growth in abdominal cavity resembled the growth of ovarian cancer in humans; the death that arose from disease complications was also consistent with human disease (28). Previously, we successfully applied surgery and PDT on tumor-bearing Fischer 344 rats using an alternative photosensitizer (hematoporphyrin monomethyl ether) to prolong the median follow-up time (29). In the present study, the immune system of Fischer 344 rats was efficient, and only four rats died after surgery and/or PDT treatment. In addition, the peritoneal cavity of Fischer 344 rats is large enough. It also allowed the more convenient collection of blood samples and delivery of laser light to the abdominal cavity. Therefore, we believe that Fischer 344 rats are particularly well suited for PDT experiments.

In conclusion, although there was no statistically significant difference between the survival time of the HB-DMSO and HB-PBCA-NP groups, PBCA-NP remains a promising drug delivery system for ovarian cancer PDT. PBCA-NP showed potential advantages for controlled drug release and tumor targeting, which might contribute to the efficacy of HB-based PDT. PDT combined with surgery could prolong animal survival time, which might result in a novel choice for the treatment of ovarian cancer.

\section{Acknowledgements}

The present study was supported by grants from the National Science Foundation of China (no. 81172488) and Outstanding Young Scientists Foundation of Shandong Province of China (BS2013YY035).

\section{References}

1. Feki A, Berardi P, Bellingan G, Major A, Krause KH, Petignat $P$, Zehra R, Pervaiz S and Irminger-Finger I: Dissemination of intraperitoneal ovarian cancer: Discussion of mechanisms and demonstration of lymphatic spreading in ovarian cancer model. Crit Rev Oncol Hematol 72: 1-9, 2009.

2. Bast RC Jr, Hennessy B and Mills GB: The biology of ovarian cancer: New opportunities for translation. Nat Rev Cancer 9: 415-428, 2009.

3. Huynh H, Teo CC and Soo KC: Bevacizumab and rapamycin inhibit tumor growth in peritoneal model of human ovarian cancer. Mol Cancer Ther 6: 2959-2966, 2007.

4. Armstrong A, Otvos B, Singh S and Debernardo R: Evaluation of the cost of CA-125 measurement, physical exam, and imaging in the diagnosis of recurrent ovarian cancer. Gynecol Oncol 131: 503-507, 2013.

5. Busch TM, Hahn SM, Wileyto EP, Koch CJ, Fraker DL, Zhang P, Putt M, Gleason K, Shin DB, Emanuele MJ, et al: Hypoxia and photofrin uptake in the intraperitoneal carcinomatosis and sarcomatosis of photodynamic therapy patients. Clin Cancer Res 10: 4630-4638, 2004. 
6. Hendren SK, Hahn SM, Spitz FR, Bauer TW, Rubin SC, Zhu T, Glatstein E and Fraker DL: Phase II trial of debulking surgery and photodynamic therapy for disseminated intraperitoneal tumors. Ann Surg Oncol 8: 65-71, 2001.

7. Guyon L, Lesage JC, Betrouni N and Mordon S: Development of a new illumination procedure for photodynamic therapy of the abdominal cavity. J Biomed Opt 17: 038001, 2012.

8. Canter RJ, Mick R, Kesmodel SB, Raz DJ, Spitz FR, Metz JM, Glatstein EJ, Hahn SM and Fraker DL: Intraperitoneal photodynamic therapy causes a capillary-leak syndrome. Ann Surg Oncol 10: 514-524, 2003.

9. Hahn SM, Putt ME, Metz J, Shin DB, Rickter E, Menon C, Smith D, Glatstein E, Fraker DL and Busch TM: Photofrin uptake in the tumor and normal tissues of patients receiving intraperitoneal photodynamic therapy. Clin Cancer Res 12: 5464-5470, 2006.

10. Portilho FA, Cavalcanti CE, Miranda-Vilela AL, Estevanato LL, Longo JP, Almeida Santos MF, Bocca AL, Martins OP, Simioni AR, Morais PC, et al: Antitumor activity of photodynamic therapy performed with nanospheres containing zinc-phthalocyanine. J Nanobiotechnol 11: 41, 2013.

11. Castano AP, Mroz P and Hamblin MR: Photodynamic therapy and anti-tumour immunity. Nat Rev Cancer 6: 535-545, 2006.

12. Shishkova N, Kuznetsova O and Berezov T: Photodynamic therapy for gynecological diseases and breast cancer. Cancer Biol Med 9: 9-17, 2012.

13. Agostinis P, Berg K, Cengel KA, Foster TH, Girotti AW, Gollnick SO, Hahn SM, Hamblin MR, Juzeniene A, Kessel D, et al: Photodynamic therapy of cancer: An update. CA Cancer J Clin 61: 250-281, 2011.

14. Ma G, Khan SI, Jacob MR, Tekwani BL, Li Z, Pasco DS, Walker LA and Khan IA: Antimicrobial and antileishmanial activities of hypocrellins A and B. Antimicrob Agents Chemother 48: 4450-4452, 2004.

15. Sun Y, Zheng Y, Lei WH, Zhou QX, Hou YJ, Zhang BW and Wang XS: Oxovanadium(IV) based hypocrellin B complexes with enhanced photodynamic activity. Dalton Trans 41: 651-657, 2012.

16. Maeda H: The enhanced permeability and retention (EPR) effect in tumor vasculature: The key role of tumor-selective macromolecular drug targeting. Adv Enzyme Regul 41: 189-207, 2001.

17. Yallapu MM, Jaggi M and Chauhan SC: Scope of nanotechnology in ovarian cancer therapeutics. J Ovarian Res 3: 19, 2010.

18. Kim JH, Kim YS, Park K, Lee S, Nam HY, Min KH, Jo HG, Park JH, Choi K, Jeong SY, et al: Antitumor efficacy of cisplatinloaded glycol chitosan nanoparticles in tumor-bearing mice. $\mathrm{J}$ Control Release 127: 41-49, 2008.
19. Upadhyay KK, Bhatt AN, Mishra AK, Dwarakanath BS, Jain S, Schatz C, Le Meins JF, Farooque A, Chandraiah G, Jain AK, et al: The intracellular drug delivery and anti tumor activity of doxorubicin loaded poly(gamma-benzyl L-glutamate)-bhyaluronan polymersomes. Biomaterials 31: 2882-2892, 2010.

20. Zhu Z, Li Y, Li X, Li R, Jia Z, Liu B, Guo W, Wu W and Jiang X: Paclitaxel-loaded poly( $N$-vinylpyrrolidone)- $b$-poly( $\varepsilon$ caprolactone) nanoparticles: Preparation and antitumor activity in vivo. J Control Release 142: 438-446, 2010.

21. Yordanov G, Evangelatov A and Skrobanska R: Epirubicin loaded to pre-polymerized poly(butyl cyanoacrylate) nanoparticles: Preparation and in vitro evaluation in human lung adenocarcinoma cells. Colloids Surf B Biointerfaces 107: 115-123, 2013.

22. Duan J, Zhang Y, Han S, Chen Y, Li B, Liao M, Chen W, Deng X, Zhao J and Huang B: Synthesis and in vitro/in vivo anti-cancer evaluation of curcumin-loaded chitosan/poly(butyl cyanoacrylate) nanoparticles. Int J Pharm 400: 211-220, 2010.

23. Muñoz-Casares FC, Rufián S, Arjona-Sánchez Á, Rubio MJ, Díaz R, Casado Á, Naranjo Á, Díaz-Iglesias CJ, Ortega R, Muñoz-Villanueva MC, et al: Neoadjuvant intraperitoneal chemotherapy with paclitaxel for the radical surgical treatment of peritoneal carcinomatosis in ovarian cancer: A prospective pilot study. Cancer Chemother Pharmacol 68: 267-274, 2011.

24. Mroz P, Xia Y, Asanuma D, Konopko A, Zhiyentayev T, Huang YY, Sharma SK, Dai T, Khan UJ, Wharton T, et al: Intraperitoneal photodynamic therapy mediated by a fullerene in a mouse model of abdominal dissemination of colon adenocarcinoma. Nanomedicine 7: 965-974, 2011.

25. DeLaney TF, Sindelar WF, Tochner Z, Smith PD, Friauf WS, Thomas G, Dachowski L, Cole JW, Steinberg SM and Glatstein E: Phase I study of debulking surgery and photodynamic therapy for disseminated intraperitoneal tumors. Int J Radiat Oncol Biol Phys 25: 445-457, 1993.

26. Song K, Kong B, Qu X, Li L and Yang Q: Phototoxicity of Hemoporfin to ovarian cancer. Biochem Biophys Res Commun 337: 127-132, 2005.

27. Seshadri M, Bellnier DA, Vaughan LA, Spernyak JA, Mazurchuk R, Foster TH and Henderson BW: Light delivery over extended time periods enhances the effectiveness of photodynamic therapy. Clin Cancer Res 14: 2796-2805, 2008.

28. Rose GS, Tocco LM, Granger GA, DiSaia PJ, Hamilton TC, Santin AD and Hiserodt JC: Development and characterization of a clinically useful animal model of epithelial ovarian cancer in the Fischer 344 rat. Am J Obstet Gynecol 175: 593-599, 1996.

29. Song K, Kong B, Li L, Yang Q, Wei Y and Qu X: Intraperitoneal photodynamic therapy for an ovarian cancer ascite model in Fischer 344 rat using hematoporphyrin monomethyl ether. Cancer Sci 98: 1959-1964, 2007. 